1

2

3

4

5

6

7

8

\section{Storage length and temperature influence infectivity and spore yield of two common Daphnia parasites}

Meghan A. Duffy* and Katherine K. Hunsberger

Department of Ecology \& Evolutionary Biology

University of Michigan

Ann Arbor, MI 48109

*Correspondence: duffymeg@umich.edu

Running head: Effects of storage on Daphnia parasites

Keywords: Metschnikowia; Pasteuria; refrigerator; freezer; burst size; spore; fitness; Daphnia dentifera 


\section{Abstract}

Daphnia and their parasites have emerged as a model system for understanding the

24 ecology and evolution of infectious diseases. Two of the most commonly studied Daphnia

25 parasites are the bacterium Pasteuria ramosa and the fungus Metschnikowia bicuspidata. In

26 addition to being the focus of numerous field studies, these two parasites have been used in many

27 laboratory experiments. However, there is little information in the scientific literature about how

28 the conditions under which these parasites are stored influence the infectivity and yield of

29 transmission stages ("spores"). This is problematic because such information is critical for

30 experiment design and data interpretation.

We tested the influence of storage length (eight treatments ranging from 1 day to 1 year)

32 and temperature $\left(-20^{\circ} \mathrm{C}\right.$ (freezer) vs. $4^{\circ} \mathrm{C}$ (refrigerator)) on spore infectivity and yield. We found

33 that Pasteuria spores survived well at both $-20^{\circ} \mathrm{C}$ and $4^{\circ} \mathrm{C}$, and remained infective even after

34 storage for one year. However, Pasteuria spore yields dropped over time, particularly at $4^{\circ} \mathrm{C}$. In

35 contrast, Metschnikowia spores were killed within days at $-20^{\circ} \mathrm{C}$. At $4^{\circ} \mathrm{C}$, Metschnikowia

36 infectivity declined steadily over a period of two months and, by four months, spores were no

37 longer infective. Spore yield from Metschnikowia-infected hosts was not significantly impacted

38 by storage length, but trended downwards.

41 that it is killed by freezer storage and that, even if it is stored in the refrigerator, infectivity

42 declines within a few months. These results might have implications for parasite distributions in

43 the field; for example, the high sensitivity of Metschnikowia to freezing might help explain why

44 it tends to be more common in deep lakes than in ponds or rock pools. 


\section{Introduction}

Studies of Daphnia-parasite interactions date back to at least the 1880s (Metchnikoff

$471884,1888,1889)$. In the past 25 years, Daphnia and their parasites have emerged as a model

48 system for understanding the ecology and evolution of infectious diseases (Ebert 2005, Lampert

49 2011, Cáceres et al. 2014). One strength of the Daphnia-parasite system is its utility in

50 laboratory experiments, which have helped uncover factors that are relevant to the ecology and

51 evolution of infectious diseases (Ebert 2008, Ebert 2011, Lampert 2011).

52 Despite being an important study system, there is surprisingly little information in the

53 scientific literature about how lab conditions, including storage length and temperature, influence

54 transmission stages of common Daphnia parasites. These transmission stages (hereafter:

55 "spores") are the infective stage of the parasite, enabling the parasite to move between host

56 individuals. If lab storage conditions influence the infectivity and/or yield of parasite spores, that

57 would affect the likelihood of an experiment succeeding. Moreover, since scientists often use

58 infectivity and spore yield as proxies for parasite fitness, impacts of lab storage on spores could

59 also affect estimates of parasite fitness.

60 Thus, understanding whether lab conditions alter spore infectivity and yield is critical for

61 experimental design. For example, we recently carried out an experiment in which we collected

62 spores of the common bacterial parasite, Pasteuria ramosa, from multiple lakes and had to store

63 them in the lab for a year before using them in experiments. We knew that Pasteuria spores

64 could remain viable in the lab for this length of time (Ebert 2005, Little et al. 2006), but had no

65 information on whether - to maximize future infectivity and spore yield - it would be better to

66 store them in the refrigerator $\left(-4^{\circ} \mathrm{C}\right)$ or freezer $\left(-20^{\circ} \mathrm{C}\right)$. For a second experiment, we wanted to

67 kill spores of the common fungal parasite, Metschnikowia bicuspidata, without killing Pasteuria 
68 spores. We knew that Pasteuria spores remain viable in the freezer (Ebert 2005, Little et al.

69 2006), even after passing through a Daphnia gut (King et al. 2013). However, we did not have

70 corresponding data on Metschnikowia, though, based on anecdotal observations, we suspected

71 Metschnikowia spores would not remain viable in the freezer. Our two studies showed that we

72 need more information regarding spore storage conditions to make informed decisions about

73 appropriate experimental design.

We tested the effects of storage length and temperature on two common Daphnia

75 parasites: Pasteuria ramosa and Metschnikowia bicuspidata. We stored spores for lengths

76 ranging from 1 day to 1 year and at two temperatures $\left(-20^{\circ} \mathrm{C}\right.$ (freezer) and $4^{\circ} \mathrm{C}$ (refrigerator)),

77 then assessed both their infectivity and the spore yield from infected hosts. This information will

78 assist other scientists interested in carrying out experiments with these model systems, and might

79 also help explain patterns of disease in natural populations.

\section{Methods}

82 We tested the effects of storage length and temperature on infectivity of spores of the

83 bacterium Pasteuria ramosa ("G/18" isolate) and the fungus Metschnikowia bicuspidata ("Std"

84 isolate). We used infection assays in which hosts were exposed to either Pasteuria or

85 Metschnikowia. While carrying out the experiment, one of us (KKH) noticed that the number of

86 spores per tube seemed to decrease over time. Thus, we also did a post-hoc analysis to determine

87 whether spore yield varied with storage length and/or temperature.

Our general procedure involved: 1) collecting $D$. dentifera that were heavily infected

89 with either Pasteuria or Metschnikowia and putting them in microcentrifuge tubes (6-9

90 individuals per tube); 2) assigning each tube to a particular storage length $\mathrm{x}$ storage temperature 
91 treatment; 3) after the appropriate storage length, removing them from storage and grinding up

92 the infected individuals in each tube; 4) using a hemocytometer to determine the density of

93 spores in each tube (our measure of spore yield); 5) adding the appropriate amount of spores

94 from a tube to a beaker containing six uninfected $D$. dentifera; 6 ) exposing the $D$. dentifera to the

95 spores for 24 hours; and, finally, 7) maintaining the exposed individuals in the lab until it was

96 possible to determine whether they were infected (our measure of infectivity).

97

(a) Impact of storage length and temperature on spore infectivity

99 We used eight storage length treatments, ranging from 1 day to 1 year (Table 1). We used

100 this range because sometimes we want to store spores longer term (as in the Pasteuria example

101 given in the introduction), whereas at other times we want to quickly kill spores (as in the

102 Metschnikowia example given in the introduction). The intermediate storage ranges were chosen

103 because, based on prior experience with these parasites, we suspected that Pasteuria would live

104 at least several months and likely a full year, whereas we suspected that Metschnikowia would

105 not survive more than a few months. We used two storage temperatures $\left(-20^{\circ} \mathrm{C}\right.$ vs. $\left.4^{\circ} \mathrm{C}\right)$, chosen

106 to correspond with common laboratory storage conditions: in the freezer or refrigerator,

107 respectively. 
Table 1. Treatment combinations for the experiment. "Pasteuria" = Pasteuria ramosa; "Metsch" = Metschnikowia bicuspidata; "wks" spore beakers and two high spore beakers per replicate tube. Full details can be found in the online repository for the data and code.

\begin{tabular}{|c|c|c|c|c|c|c|c|c|}
\hline \multirow[t]{2}{*}{ Parasite } & \multirow{2}{*}{$\begin{array}{l}\text { Storage } \\
\text { temp. } \\
\left({ }^{\circ} \mathrm{C}\right)\end{array}$} & \multirow[t]{2}{*}{$\begin{array}{l}\text { Storage } \\
\text { length }\end{array}$} & \multirow{2}{*}{$\begin{array}{c}\text { Spore } \\
\text { doses } \\
\text { (spores } / \mathrm{mL} \text { ) }\end{array}$} & \multirow{2}{*}{$\begin{array}{c}\text { \# infected } \\
\text { individuals } \\
\text { per tube }\end{array}$} & \multirow{2}{*}{$\begin{array}{c}\# \\
\text { replicate } \\
\text { tubes }\end{array}$} & \multicolumn{2}{|c|}{$\begin{array}{l}\text { \# beakers per } \\
\text { replicate tube }\end{array}$} & \multirow[t]{2}{*}{$\begin{array}{l}\text { Date of exposure } \\
\text { (month/day/year) }\end{array}$} \\
\hline & & & & & & $\begin{array}{l}\text { Low } \\
\text { spore } \\
\text { dose }\end{array}$ & $\begin{array}{l}\text { High } \\
\text { spore } \\
\text { dose }\end{array}$ & \\
\hline Pasteuria & 4 & 1 day & 500,2000 & 6 & 5 & 2 & 2 & $3 / 14 / 2017$ \\
\hline Pasteuria & 4 & 2 wks & 500,2000 & 6 & 5 & 2 & 2 & $3 / 27 / 2017$ \\
\hline Pasteuria & 4 & 4 wks & 500,2000 & 6 & 5 & $1-3$ & 2 & $4 / 10 / 2017$ \\
\hline Pasteuria & 4 & $2 \mathrm{mo}$. & 500,2000 & 6 & 5 & 2 & 2 & $5 / 30 / 2017$ \\
\hline Pasteuria & 4 & $4 \mathrm{mo}$. & 500,2000 & 6 & 5 & 2 & 2 & $7 / 11 / 2017$ \\
\hline Pasteuria & 4 & $6 \mathrm{mo}$. & 500,2000 & 6 & $4-5$ & $2-4$ & $1-2$ & $9 / 18 / 2017$ \\
\hline Pasteuria & 4 & $9 \mathrm{mo}$. & 500,2000 & 6 & $4-5$ & $2-4$ & $1-2$ & $12 / 11 / 2017$ \\
\hline Pasteuria & 4 & 1 year & 500,2000 & 6 & $4-5$ & 2 & $1-2$ & $3 / 6 / 2018$ \\
\hline Pasteuria & -20 & 1 day & 500,2000 & 6 & 5 & 2 & 2 & $3 / 14 / 2017$ \\
\hline Pasteuria & -20 & 2 wks & 500,2000 & 6 & 5 & 2 & 2 & $3 / 27 / 2017$ \\
\hline Pasteuria & -20 & 4 wks & 500,2000 & 6 & 5 & 2 & 2 & $4 / 10 / 2017$ \\
\hline Pasteuria & -20 & $2 \mathrm{mo}$ & 500,2000 & 6 & 5 & 2 & 2 & $5 / 30 / 2017$ \\
\hline Pasteuria & -20 & $4 \mathrm{mo}$. & 500,2000 & 6 & 5 & 2 & 2 & $7 / 11 / 2017$ \\
\hline Pasteuria & -20 & $6 \mathrm{mo}$. & 500,2000 & 6 & 5 & 2 & 2 & 9/18/2017 \\
\hline Pasteuria & -20 & $9 \mathrm{mo}$. & 500,2000 & 6 & $4-5$ & 2 & $2-4$ & $12 / 11 / 2017$ \\
\hline Pasteuria & -20 & 1 year & 500,2000 & 6 & 5 & 2 & 2 & $3 / 6 / 2018$ \\
\hline Metsch. & 4 & 1 day & 250,1000 & 9 & 3 & 2 & 2 & $3 / 14 / 2017$ \\
\hline Metsch. & 4 & 2 wks & 250,1000 & 9 & 3 & 2 & 2 & $3 / 27 / 2017$ \\
\hline Metsch. & 4 & 4 wks & 250,1000 & 9 & 3 & 2 & 2 & $4 / 10 / 2017$ \\
\hline Metsch. & 4 & $2 \mathrm{mo}$ & 250,1000 & 9 & 3 & 2 & 2 & $5 / 30 / 2017$ \\
\hline Metsch. & 4 & $4 \mathrm{mo}$. & 250,1000 & 9 & 3 & 2 & 2 & $7 / 11 / 2017$ \\
\hline Metsch. & 4 & $6 \mathrm{mo}$. & 250,1000 & 9 & 3 & 2 & 2 & $9 / 18 / 2017$ \\
\hline Metsch. & 4 & $9 \mathrm{mo}$. & 250,1000 & 9 & 3 & 2 & 2 & $12 / 11 / 2017$ \\
\hline Metsch. & 4 & 1 year & 250,1000 & 9 & 3 & 2 & 2 & $3 / 6 / 2018$ \\
\hline Metsch. & -20 & 1 day & 1000 & 7 & 3 & 0 & 2 & $3 / 14 / 2017$ \\
\hline Metsch. & -20 & 2 wks & 1000 & 7 & 3 & 0 & 2 & $3 / 27 / 2017$ \\
\hline
\end{tabular}


We maintain separate laboratory stock cultures of Pasteuria and Metschnikowia. We

114 collected heavily infected Daphnia dentifera from these cultures on 13 March 2017; individuals

115 were identified as heavily infected based on their increased opacity, which makes them appear

116 bright when illuminated. We put Pasteuria-infected $D$. dentifera in $1.5 \mathrm{~mL}$ microcentrifuge tubes

117 that were then randomly assigned to a particular temperature and storage length treatment. We

118 then repeated the process for Metschnikowia-infected D. dentifera. The exact number of infected 119 individuals that were added to each tube is given in Table 1.

120 For parasite exposures, we used two spore doses for each parasite, as we thought the

121 lower dose would detect treatment effects at times when infectivity remained high, while the

122 higher dose could detect differences between treatments when infectivity was low. Pilot studies

123 indicated that Metschnikowia dies rapidly when stored at $-20^{\circ} \mathrm{C}$. Therefore, for the $-20^{\circ} \mathrm{C}$

124 Metschnikowia treatment, we used only the high dose exposure as we expected to see no

125 infections at the low spore dose, and included only the two shortest storage lengths, to avoid

126 wasting spores.

127 We used a pestle (Fisherbrand Pellet Pestle with Cordless Motor) to grind infected

128 Daphnia in one of the microcentrifuge tubes (corresponding to the treatments indicated in Table

129 1) and used a hemocytometer to determine the spore density in the tube. We then generated the

130 desired spore dose by adding the appropriate volume from the tube (which contained 6-9

131 infected individuals, as indicated in Table 1) to a $150 \mathrm{~mL}$ beaker filled with $100 \mathrm{~mL}$ of filtered

132 lake water (D. dentifera survival is greatly enhanced by culturing in filtered lake water as

133 compared to an artificial medium). Each beaker contained six uninfected $D$. dentifera that were

134 six days old. We used the "Mid37" genotype, which is susceptible to both the "G/18" Pasteuria

135 isolate and the "Std" Metschnikowia isolate (Auld et al. 2014a). The number of replicate tubes is 
136 indicated in Table 1; with the exception of the Metschnikowia $-20^{\circ} \mathrm{C}$ treatment (which did not

137 have low spore beakers), a single $1.5 \mathrm{~mL}$ microcentrifuge tube was used to generate the spores

138 for low spore dose and high spore dose beakers, as indicated in Table 1 in the "\# beakers per 139 replicate tube" columns.

140 We exposed $D$. dentifera to spores at $20^{\circ} \mathrm{C}$ in 16:8 light:dark (consistent with previous

141 studies; e.g., Auld et al. 2014a). After 24 hours of spore exposure, the six D. dentifera

142 individuals in each beaker were transferred to beakers containing filtered lake water that did not 143 contain spores. Individuals were fed 10,000 cells $/ \mathrm{mL}$ of a nutritious green alga, Ankistrodesmus

144 falcatus, during parasite exposure and 20,000 cells/mL otherwise; we used a lower food dose 145 during parasite exposure because it results in higher infection levels at a given spore dose (Hall 146 et al. 2007). We added food to beakers four days/week; feeding four vs. seven days a week has

147 no impact on our ability to determine whether individuals are infected, and reduces labor

148 associated with feeding. Individuals were maintained in the lab until it was possible to determine 149 whether they had been successfully infected (approximately 28 days for Pasteuria and 10-11 150 days for Metschnikowia). Infections were diagnosed by observing individuals under a dissecting 151 microscope; as indicated earlier, infected hosts are much more opaque, with spores filling their 152 hemolymph.

We carried out two analyses of the infectivity data, both using generalized linear models

154 (glms) with a binomial error structure. First, we analyzed the Pasteuria data using a model that 155 included dose, storage temperature, storage length (in days), and their interactions as 156 independent variables, and a matrix of uninfected and infected hosts (per individual beaker) as 157 the response variable. For these analyses, we used storage length as measured in days, using the 158 exact lengths calculated from the difference between 13 March and the day of exposure given in 
159 Table 1. Second, we analyzed the data from the Metschnikowia $4{ }^{\circ} \mathrm{C}$ treatment on its own, as we

160 only had two storage lengths for the $-20^{\circ} \mathrm{C}$ treatment. This model included dose, storage length

161 (in days), and their interaction as independent variables, and a matrix of uninfected and infected

162 hosts (per individual beaker) as the response variable. In some cases, the number of days did not

163 precisely match to the treatment label (in months or years) due to logistical constraints (e.g., the

164 "1 year" treatment was actually 358 days). The exact treatment lengths were as follows: 1 day (1 165 day), 2 weeks (14 days), 4 weeks (28 days), 2 months (78 days), 4 months (120 days), 6 months

166 (189 days), 9 months (273 days), 1 year (358 days).

167

(b) Variation in spore yield over time

169 We also used glms to analyze the spore yield data, which was obtained with the

170 hemocytometer counts described above. We analyzed data on Pasteuria spore yield using a

171 model that included dose, storage length (in days), storage temperature, and their interactions.

172 For the analysis of Metschnikowia spore yield in the $4^{\circ} \mathrm{C}$ treatment, we used a model that

173 included dose, storage length (in days), and their interaction as independent variables. For both

174 analyses, the dependent variable was $\ln$ (spores per infected Daphnia).

175 All analyses were done in R 3.5.0. All data and code can be found on github:

176 https://github.com/duffymeg/sporestorage.

\section{Results}

179 Pasteuria spores remained infective even after 1 year of storage (Figure 1; Table 2). Post-

180 hoc analyses indicate that the increase in infectivity with storage length was significant for the

181 high spore treatment at both temperatures, and for the low spore treatment at $4{ }^{\circ} \mathrm{C}$. 
Infectivity of Pasteuria spores stored at $-20^{\circ} \mathrm{C}$ and $4^{\circ} \mathrm{C}$

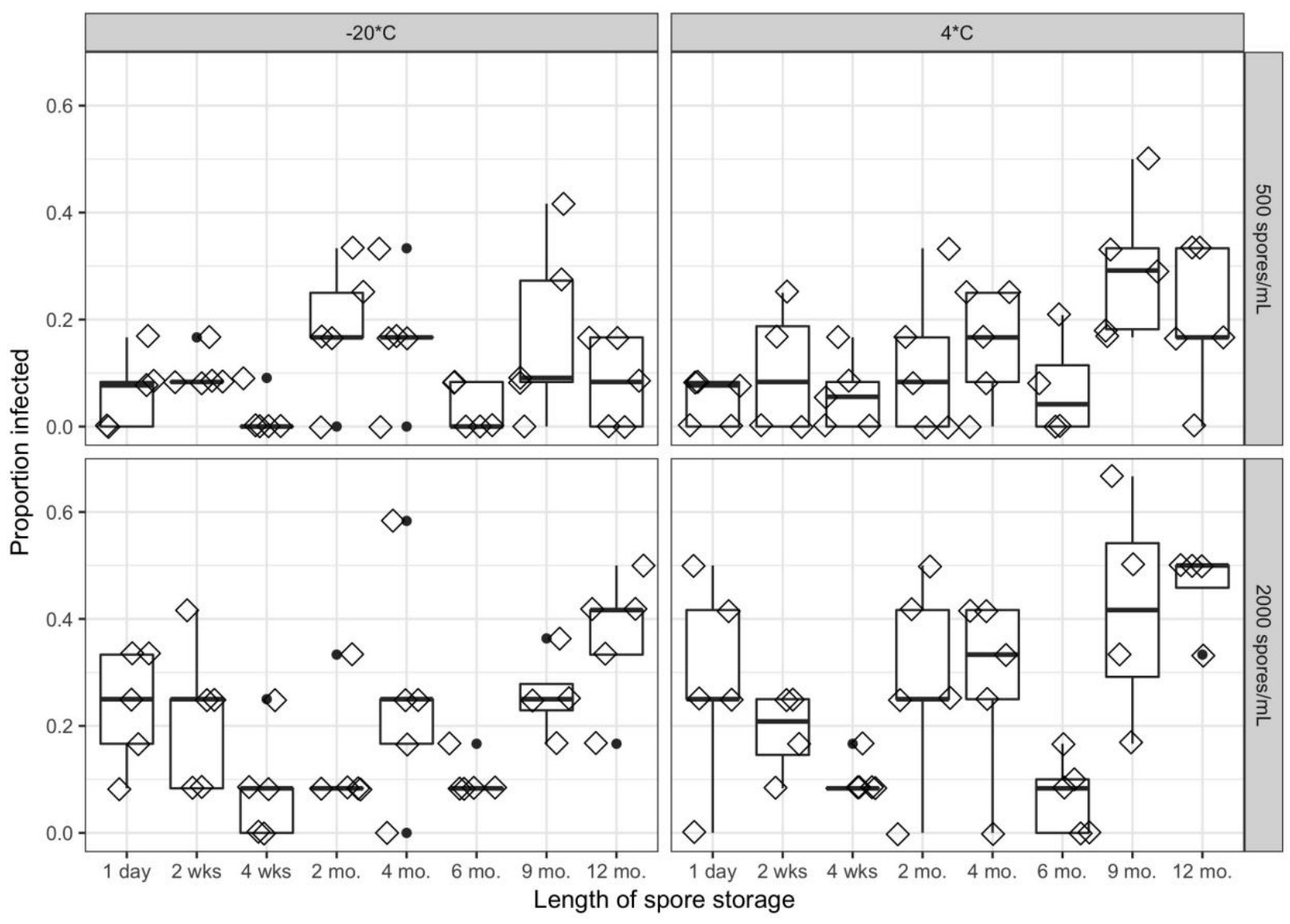

184 Figure 1. Spores of the bacterium Pasteuria ramosa remained infective even after a year in

185 storage. Top row: Low spore dose treatment (500 spores/mL); bottom: high spore dose treatment

186 (2000 spores $/ \mathrm{mL}$ ). Left: $-20^{\circ} \mathrm{C}$ treatment (freezer); right: $4^{\circ} \mathrm{C}$ treatment (refrigerator). Spores

187 remained infective in all of these treatments. Post-hoc analyses indicated that infectivity

188 increased in the high spore treatment at both temperatures and in the low spore treatment at $4^{\circ} \mathrm{C}$

$189(p<0.015$ in all three treatments). 
192 Table 2. Results of statistical analysis of factors influencing infectivity of Pasteuria spores.

\begin{tabular}{|l|c|c|}
\hline Effect & $\mathbf{Z}$ & $\boldsymbol{p}$ \\
\hline Storage temperature & -0.63 & 0.529 \\
\hline Storage length & 0.83 & 0.408 \\
\hline Dose & 2.06 & $\mathbf{0 . 0 4 0}$ \\
\hline Storage temperature $*$ storage length & 2.01 & $\mathbf{0 . 0 4 4}$ \\
\hline Storage temperature $*$ dose & 1.10 & 0.273 \\
\hline Storage length $*$ dose & 0.93 & 0.353 \\
\hline Storage temperature $*$ storage length $*$ dose & -1.43 & 0.153 \\
\hline
\end{tabular}

195 at $4^{\circ} \mathrm{C}$ (Figure 2; storage length: $\mathrm{t}=-2.84, p=0.006$; storage temperature: $\mathrm{t}=-2.04, p=0.045$;

196 interaction: $\mathrm{t}=-2.50, p=0.014)$. Even in the $-20^{\circ} \mathrm{C}$ treatment, there was a significant drop in

197 spore yield with increased storage length $(\mathrm{t}=-2.69, p=0.011)$. It is possible that the increase in 198 infectivity seen above (Figure 1) and the drop in spore yield (Figure 2) are both explained by 199 non-viable spores degrading over time to the point where we could not visually identify them as 200 spores. 


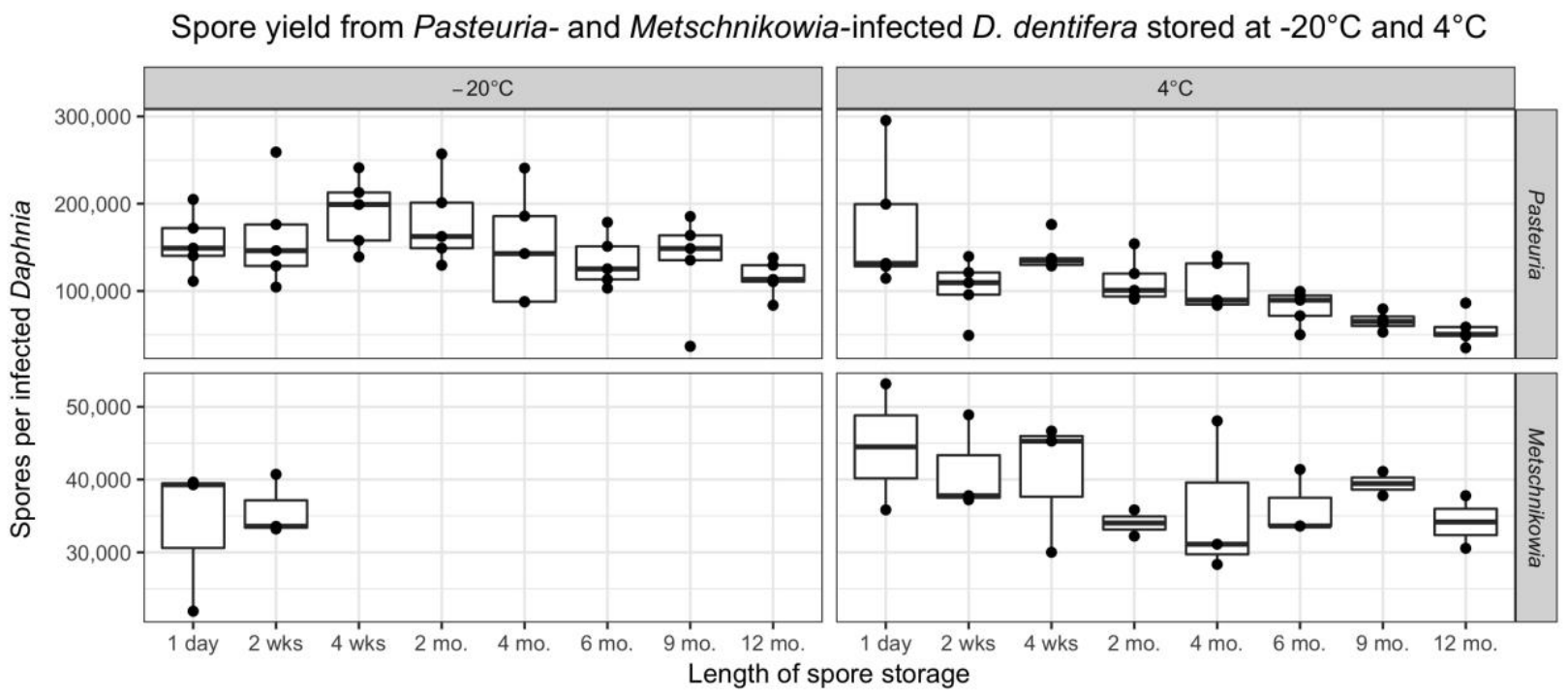

204 Figure 2. Spore yield from Pasteuria-infected hosts (top row) declined with increasing storage at $4^{\circ} \mathrm{C}$ (bottom right panel) did not change significantly over time, but trended downwards. one individual became infected out of the 36 that were exposed to a high dose of Metschnikowia

211 spores that had been stored at $-20^{\circ} \mathrm{C}$ for 24 hours; in contrast, 17 of the 36 individuals exposed to

212 spores that had been stored at $4^{\circ} \mathrm{C}$ for 24 hours became infected. No individuals became infected

213 when exposed to Metschnikowia spores that had been stored at $-20^{\circ} \mathrm{C}$ for two weeks. The

214 infectivity of Metschnikowia spores that had been stored at $4^{\circ} \mathrm{C}$ declined over the first two

215 months of storage and, by four months, spores were no longer infective. In our analysis of the

216 Metschnikowia $4^{\circ} \mathrm{C}$ treatment data, only storage length significantly impacted infectivity (dose: $\mathrm{z}$

$217=0.280, p=0.780$; storage length: $\mathrm{z}=-3.58, p=0.0003$; interaction: $\mathrm{z}=0.840, p=0.401)$. 


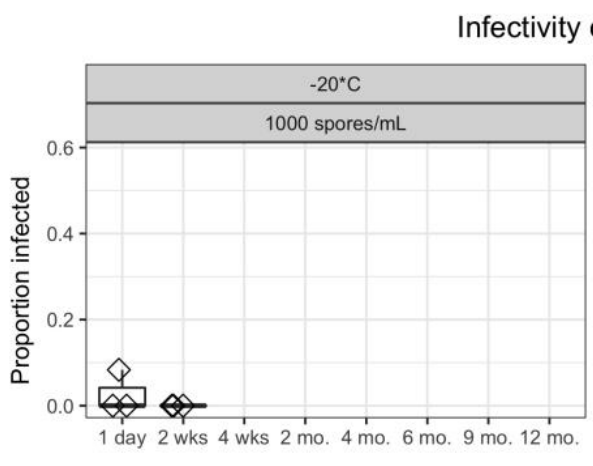

Infectivity of Metschnikowia spores stored at $-20^{\circ} \mathrm{C}$ and $4^{\circ} \mathrm{C}$

220 Figure 3. Spores of the fungus Metschnikowia bicuspidata rapidly lost infectivity.

221 Metschnikowia spores were killed within days at $-20^{\circ} \mathrm{C}$ (left panel) and infectivity declined

222 sharply over 2 months at $4^{\circ} \mathrm{C}$ (center and right panels).

At $4^{\circ} \mathrm{C}$, spore yield from Metschnikowia-infected hosts did not change significantly with

storage length $(\mathrm{t}=-1.25, p=0.229$; Figure 2$)$. However, the trend was similar to that in

Pasteuria-infected hosts (Figure 2), and the lack of significance may simply reflect lower power based on having only three replicate tubes for Metschnikowia vs. five for Pasteuria (Table 1).

\section{Discussion}

Infectivity and spore yield of two commonly studied Daphnia parasites were strongly

231 influenced by storage length and temperature. Spores of the bacterium Pasteuria ramosa

232 survived and remained infective for at least a year in both the refrigerator and the freezer.

233 However, spore yield from Pasteuria-infected hosts unexpectedly declined over time, especially

234 in the refrigerator. Spores of the fungus Metschnikowia bicuspidata, on the other hand, were

235 rapidly killed by freezing; when stored in the refrigerator, Metschnikowia infectivity declined

236 sharply over the first two months of storage and, by four months, spores were no longer 
237 infective. Spore yield of Metschnikowia-infected hosts trended downwards, but was not

238 significantly impacted by storage length. Thus, when using these parasites in lab experiments,

239 protocols should take into account the effects of storage length and temperature on spore

240 infectivity and yield.

242 important implications for experimental design (Figures 1 and 3). While Pasteuria spores can be

243 stored in the lab for relatively long periods (months to years) before being used to infect hosts,

244 Metschnikowia spores lose their infectivity over a period of weeks to months. Because of this,

245 our lab always seeks to use Metschnikowia spores that are less than one month old for

246 experiments. This presents challenges for experiments exposing field-collected hosts to field-

247 collected spores. Such experiments require growing enough individuals of each host genotype in

248 the lab prior to spore exposure. Ideally, these hosts would be grown for several generations under

249 standardized conditions to control for maternal effects (i.e., influences of the mother's

250 environment on an offspring's phenotype). However, experiments with field-collected hosts and

251 spores must trade leaving more time to grow more host individuals against the rapid loss of

252 infectivity of the field-collected spores.

254 the environment between epidemic outbreaks. Metschnikowia dynamics in lake populations are

255 strongly seasonal, with disease outbreaks occurring in late summer and autumn (Duffy et al.

256 2009). Moreover, in the Daphnia-Metschnikowia system that has been best studied, the host,

257 Daphnia dentifera, is dormant in the sediment for several months of the year. Thus,

258 Metschnikowia is presumably able to remain infective in sediments longer than in

259 microcentrifuge tubes in the lab. Indeed, it is possible to take sediment from a lake that had an 
260 epidemic in a previous year and use it to start epidemics in whole-water column enclosures in a

261 lake that did not have an epidemic (A.J. Tessier, personal communication).

263 populations. While Metschnikowia has been found in ponds (Green 1974, Codreanu \&

264 Codreanu-Balcescu 1981), its prevalence in those habitats tends to be low (<4\%) (Stirnadel \&

265 Ebert 1997, Goren \& Ben-Ami 2013). In stratified lakes, on the other hand, Metschnikowia

266 prevalence can be quite high, reaching 20\% - 40\% (Hall et al. 2011, Strauss et al. 2016).

267 Metschnikowia's intolerance of freezing might be a factor driving this pattern, as the sediments

268 in ponds are much more likely to freeze. However, it is likely that other factors — such as

269 different predation environments and stratification itself - also influence the relative prevalence

270 in lakes vs. ponds (Auld et al. 2014a).

272 result, is frequently measured in studies of these two parasites (e.g., Jensen et al. 2006, Duffy et

273 al. 2011, Auld et al. 2014a, Auld et al. 2014b). Our results suggest that the spore yield from

274 infected hosts decreases over time, especially if the hosts are stored in the refrigerator (Figure 2).

275 Ideally, spore counts from infected hosts should be done as soon after collection as possible.

276 However, if spore counts cannot be done quickly (within days to weeks), our results suggest that

277 samples should be stored in the freezer until they can be counted to reduce spore loss (for

278 Metschnikowia, this should only be done if the spores are not needed for future infections). Even

279 with freezer storage, spore yield will decline over time, so it is especially important to intersperse 280 individuals from different treatments when counting samples, rather than counting all individuals

281 from a single treatment before moving on to the next. 
Our experiment used one isolate of Pasteuria and one isolate of Metschnikowia. It is

283 possible that genotypes differ in their ability to tolerate storage under lab (and field) conditions.

284 We hypothesize that such variation is more likely in Pasteuria than in Metschnikowia, since

285 Pasteuria shows substantial variation (Carius et al. 2001, Mouton \& Ebert 2008, Auld et al.

286 2012), whereas Metschnikowia shows strikingly little phenotypic and genotypic variation (Duffy

287 \& Sivars-Becker 2007, Wolinska et al. 2009, Searle et al. 2015). However, whether either

288 parasite contains genetic variation related to tolerance of storage conditions remains to be tested.

Daphnia and their microparasites have emerged as an important study system for

290 understanding the ecology and evolution of infectious diseases (Ebert 2005, Lampert 2011,

291 Cáceres et al. 2014). Our study provides valuable information on how storage length and

292 temperature influence spore infectivity and yield from infected hosts. When designing

293 experiments, scientists should take into account that spore yield of Pasteuria declines over time,

294 especially at $4{ }^{\circ} \mathrm{C}$, and that Metschnikowia spores are killed rapidly at $-20^{\circ} \mathrm{C}$ and die within

295 weeks to months at $4^{\circ} \mathrm{C}$.

296

297 Acknowledgments

298 This work was supported by the US National Science Foundation (DEB-1353806). We thank

299 Carla Cáceres for helpful discussions and Sarah Boon for comments on this manuscript.

\section{References}

302 Auld SK, Hall SR, Ochs JH, Sebastian M, Duffy MA (2014a) Predators and patterns of within- 
Auld SKJR, Hall SR, Duffy MA (2012) Epidemiology of a Daphnia-multiparasite system and its implications for the Red Queen. PLoS ONE 7:e39564

Auld SKJR, Wilson PJ, Little TJ (2014b) Rapid change in parasite infection traits over the course of an epidemic in a wild host-parasite population. Oikos 123:232-238

Cáceres CE, Tessier AJ, Duffy MA, Hall SR (2014) Disease in freshwater zooplankton: what have we learned and where are we going? Journal of Plankton Research 36:326-333

Carius HJ, Little TJ, Ebert D (2001) Genetic variation in a host-parasite association: Potential for coevolution and frequency-dependent selection. Evolution 55:1136-1145

Codreanu R, Codreanu-Balcescu D (1981) On two Metschnikowia yeast species producing hemocoelic infections in Daphnia magna and Artemia salina (Crustacia, Phyllopoda) from Romania. Journal of Invertebrate Pathology 37:22-27

Duffy MA, Hall SR, Cáceres CE, Ives AR (2009) Rapid evolution, seasonality and the termination of parasite epidemics. Ecology 90:1441-1448

Duffy MA, Housley JM, Penczykowski RM, Cáceres CE, Hall SR (2011) Unhealthy herds: indirect effects of predators enhance two drivers of disease spread. Functional Ecology

Duffy MA, Sivars-Becker L (2007) Rapid evolution and ecological host-parasite dynamics. $25: 945-953$

Ebert D (2005) Ecology, epidemiology and evolution of parasitism in Daphnia, Vol. National Library of Medicine (US), National Center for Biotechnology Information, Bethesda, MD Curr Opin Microbiol 11:290-301 
328 Ebert D (2011) A genome for the environment. Science 331:539-540

329 Goren L, Ben-Ami F (2013) Ecological correlates between cladocerans and their endoparasites from permanent and rain pools: patterns in community composition and diversity.

Green J (1974) Parasites and epibionts of Cladocera. Transactions of the Zoological Society of London 32:417-515

Hall SR, Becker CR, Duffy MA, Cáceres CE (2011) Epidemic size determines population-level effects of parasites. Oecologia 166:833-842

Hall SR, Sivars-Becker L, Becker C, Duffy MA, Tessier AJ, Cáceres CE (2007) Eating yourself sick: transmission of disease as a function of feeding biology of hosts. Ecology Letters

Jensen KH, Little T, Skorping A, Ebert D (2006) Empirical support for optimal virulence in a castrating parasite. Plos Biology 4:1265-1269

King KC, Auld S, Wilson PJ, James J, Little TJ (2013) The bacterial parasite Pasteuria ramosa is not killed if it fails to infect: implications for coevolution. Ecol Evol 3:197-203

Little TJ, Watt K, Ebert D (2006) Parasite-host specificity: Experimental studies on the basis of parasite adaptation. Evolution 60:31-38

347 Metchnikoff E (1884) Uber eine sprosspilzkrankheit der daphnien; beitrag zur lehre uber den kampf der phagozyten gegen krankheitserreger. Virch Arch 96:177-195

Metchnikoff E (1888) Pasteuria ramosa un representant des bacteries a division longitudinale. 
351 Metchnikoff E (1889) Contributions à l'etude du pléomorphisme des bactériens. Annales de

$352 \quad$ l'Institut Pasteur 3:165-170

353 Mouton L, Ebert D (2008) Variable-number-of-tandem-repeats analysis of genetic diversity in

$354 \quad$ Pasteuria ramosa. Curr Microbiol 56:447-452

355 Searle CL, Ochs JH, Cáceres CE, Chiang SL, Gerardo NM, Hall SR, Duffy MA (2015)

356 Plasticity, not genetic variation, drives infection success of a fungal parasite. Parasitology

$357 \quad 142: 839-848$

358 Stirnadel HA, Ebert D (1997) Prevalence, host specificity and impact on host fecundity of

359 microparasites and epibionts in three sympatric Daphnia species. Journal of Animal

$360 \quad$ Ecology 66:212-222

361 Strauss AT, Shocket MS, Civitello DJ, Hite JL, Penczykowski RM, Duffy MA, Cáceres CE, Hall

362 SR (2016) Habitat, predators, and hosts regulate disease in Daphnia through direct and

363 indirect pathways. Ecological Monographs 86:393-411

364 Wolinska J, Giessler S, Koerner H (2009) Molecular identification and hidden diversity of novel

365 Daphnia parasites from European lakes. Appl Environ Microbiol 75:7051-7059 